\title{
Etude d'un absorbeur de houle de type persiennes
}

\section{Daniel Caminade}

Maître de Conférence, $L M F-G C$, Université du Havre

\author{
Alexandre Arsié \\ Doctorant, LMF-GC, Université du Havre
}

\section{Résumé}

Les dispositifs amortisseurs de houle pour les installations de laboratoire doivent être d'une grande efficacité et prendre peu de place. Le système à persiennes testé entre dans la categorie des digues verticales à paroi perméable et permet d'atteindre des coefficients de réflexion d'environ $35 \%$, ce qui est honorable pour une structure de génie civil, mais doit être amélioré pour des installations de laboratoire.

\section{Introduction}

Les essais sur modèles réduits physiques permettent d'étudier de manière commode l'action de la houle sur les ouvrages de génie civil. La recherche de dispositifs permettant de limiter les phénomènes parasites, tels que la réflexion de l'onde incidente en fond de canal à houle ou le long des parois d'une cuve à houle, est un souci constant des expérimentateurs.

C'est ainsi que, dans le cadre de la mise au point de la «petite cuve » à houle pour la Direction des Constructions Navales au Val de Reuil, nous avons été amenés à tester un dispositif d'amortissement de la houle de type persiennes.

Le but de ces essais était de tester un éventail de cas aussi large que possible de façon à évaluer globalement l'efficacité d'un tel dispositif.

\section{Description du dispositif expérimental}

\subsection{Equipements généraux}

Les essais ont été conduits dans un canal à houle de 23 mètres de long, de 0,80 mètre de large et de hauteur utile, équipé d'un batteur plan à mouvement horizontal capable de générer de la houle régulière.

La mesure de la houle a été réalisée grâce à des sondes résistives. Les performances du dispositif (coefficient de réflexion) ont été évaluées par la méthode des ventres et noeuds. 


\subsection{Le dispositif persiennes}

Le dispositif comprend :

Une paroi amon perméable faite de lattes en P.V.C. fixées sur des montant transparents en Plexiglas.

Une paroi étanche qui représente le mur de la cuve (ou qui pourrait représenter la face arrière d'un caisson).

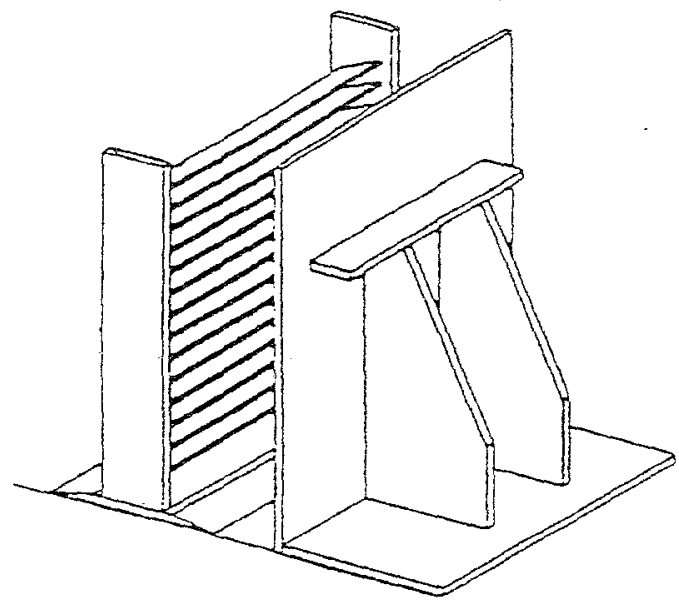

Figure I : Schéma de principe du dispositif persiennes

Les dispositions constructives permettent de faire varier:

- l'inclinaison des lattes de la paroi amont.

- l'écartement des lattes.

- la largeur des lattes.

- la porosité de la latte.

et bien entendu...

- la dimension de la chambre d'expansion de la houle comprise entre les deux parois (dimension rinesurée entre la paroi opaque et l'axe de la paroi perméable).

\subsection{Les paramètres hydrauliques}

La hauteur d'eau dans le canal a été fixée à 0,40 mètre pour pouvoir aisément et sans conversion d'échelle, comparer les résultats avec d'autres essais réalisés au L.M.F.G.C. et notamment sur les caissons Jarlan.

Les périodes de la houle ont été fixées entre 0,5 et 2,4 s. pour obtenir un balayage des périodes utilisées dans les installations d'essai.

Les creux de la houle incidente ont été limités soit par le déferlement soit par les caractéristiques de l'installation (batteur et filtres). On a pu en général obtenir des creux de l'ordre de $10 \mathrm{~cm}$. 


\subsection{Les configurations testées.}

Trois types de configurations ont été testées :

- Lattes espacées de $5 \mathrm{~cm}$ et inclinées de $20 \%$ associées à des chambres de 20 et $40 \mathrm{~cm}$ de large. et $80 \mathrm{~cm}$.

- Lattes espacées de $5 \mathrm{~cm}$ et inclinées de $40 \%$ avec des chambres de 40,60 de $60 \mathrm{~cm}$.

-Lattes alternativement horizontales et inclinées de $40 \%$ pour une chambre
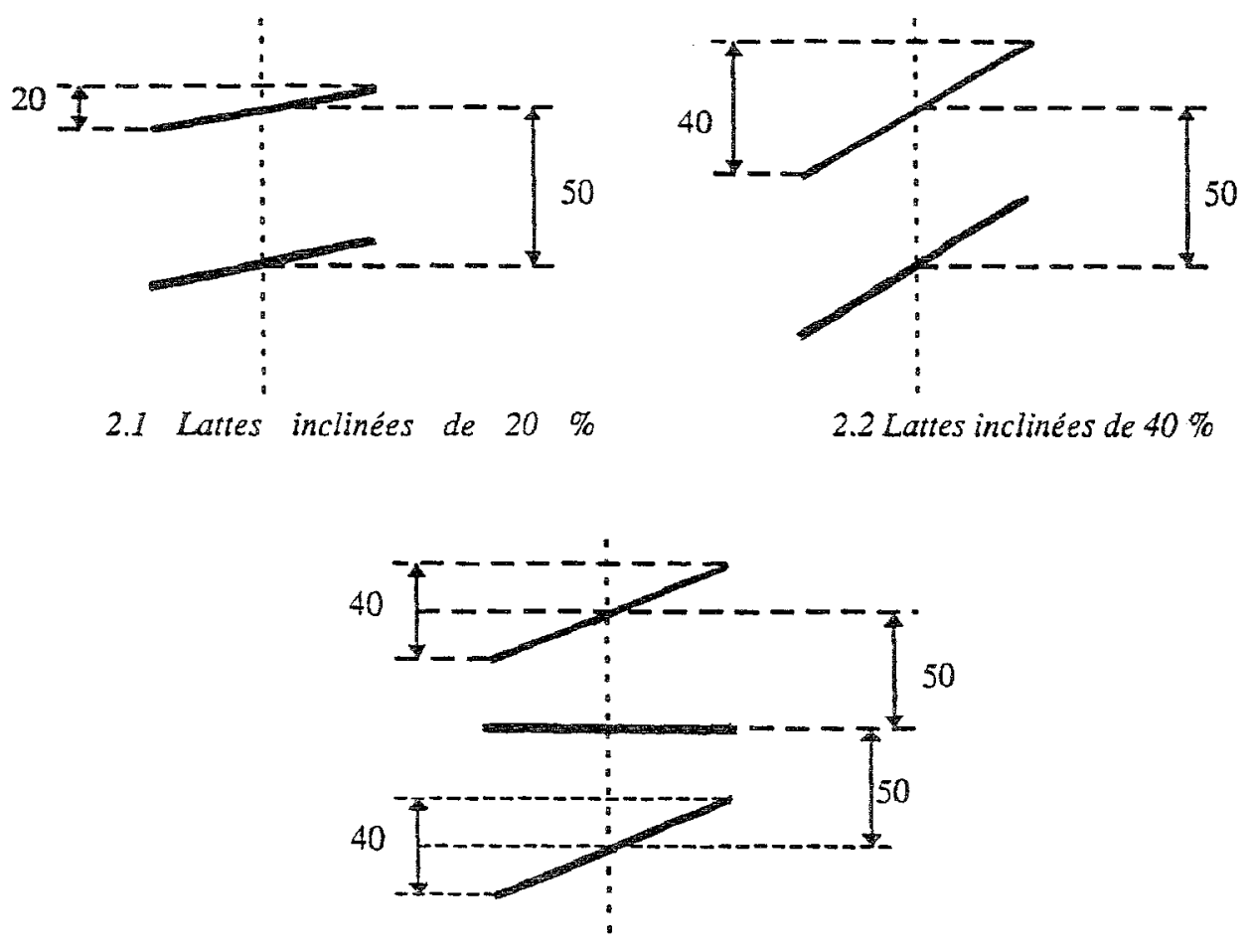

2.3 Lattes alternées

Figure 2 : Schéma des configurations testées

\section{Les résultats}

Les résultats présentés ici, portent sur l'analyse du coefficient de réflexion de la structure en fonction de la cambrure de la houle (H/L : creux incident divisé par la longueur d'onde) qui apparait comme un paramètre fondamental permettant de regrouper les résultats obtenus pour les diverses périodes. 


\subsection{Lattes inclinées de $20 \%$}

Dans ce type de configuration la porosité apparente en vue de face est de $40 \%$, du même ordre de grandeur que celle représentée par la face amont du caisson Jarlan.

Les résultats obtenus sont assez médiocres car la paroi amont se comporte comme pratiquement totalement perméable : les coefficients de réflexion sont de l'ordre de 60 à $80 \%$ sauf pour la période de $0.86 \mathrm{~s}$. avec des cambrures supérieures à $4 \%$ et pour une largeur de chambre de $40 \mathrm{~cm}$ pour laquelle on obtient des coefficients de l'ordre de $45 \%$.

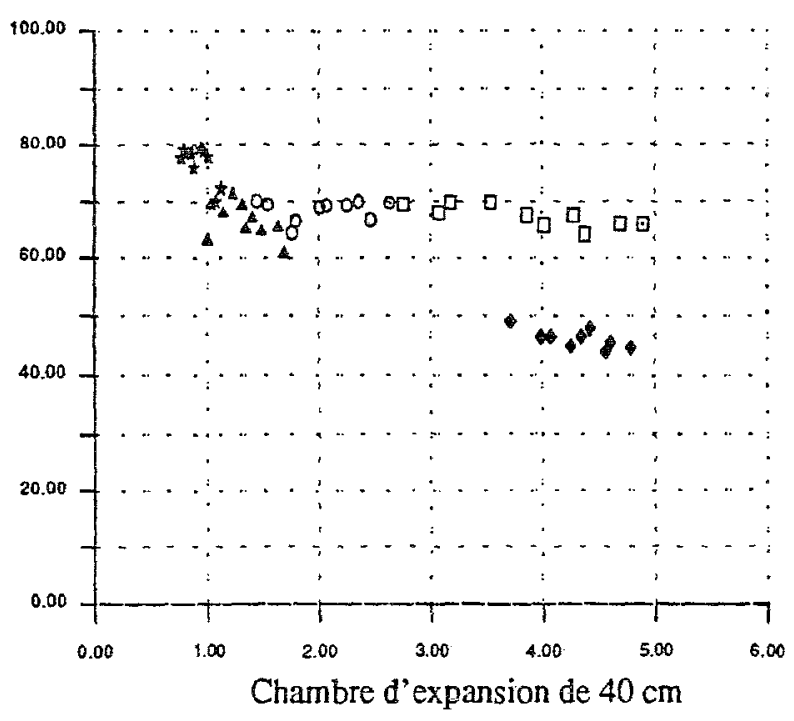

\begin{tabular}{|llll|}
\hline $\mathrm{T}=0,86 \mathrm{~s}$ & $\star$ & $\mathrm{T}=1,6 \mathrm{~s}$ & 0 \\
$\mathrm{~T}=1,1 \mathrm{~s}$ & $\square$ & $\mathrm{T} 1,9 \mathrm{~s}$ & $\star$ \\
$\mathrm{T} 2,4 \mathrm{~s}$ & $\star$ & & \\
\hline
\end{tabular}

Figure 3 : Evolution du coefficient de réflexion $\mathrm{Kr}$ en fonction de la cambrure $\gamma$

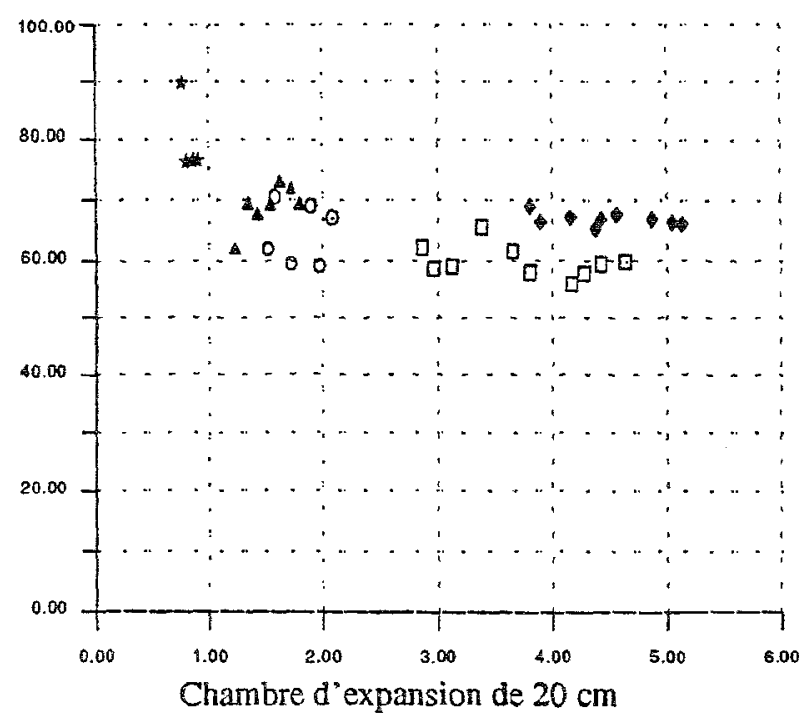




\subsection{Lattes inclinées à $40 \%$}

Les résultats précédents nous ont conduits à :

-incliner deux fois plus les lattes des persiennes.

-sélectionner des largeurs de chambres importantes $(40,60$ er $80 \mathrm{~cm})$

Les résultats font l'objet des graphiques de la figure 4 et montrent que pour des cambrures qui n'excèdent pas $5,5 \%$ le coefficient de réflexion décroît en fonction de la cambrure.

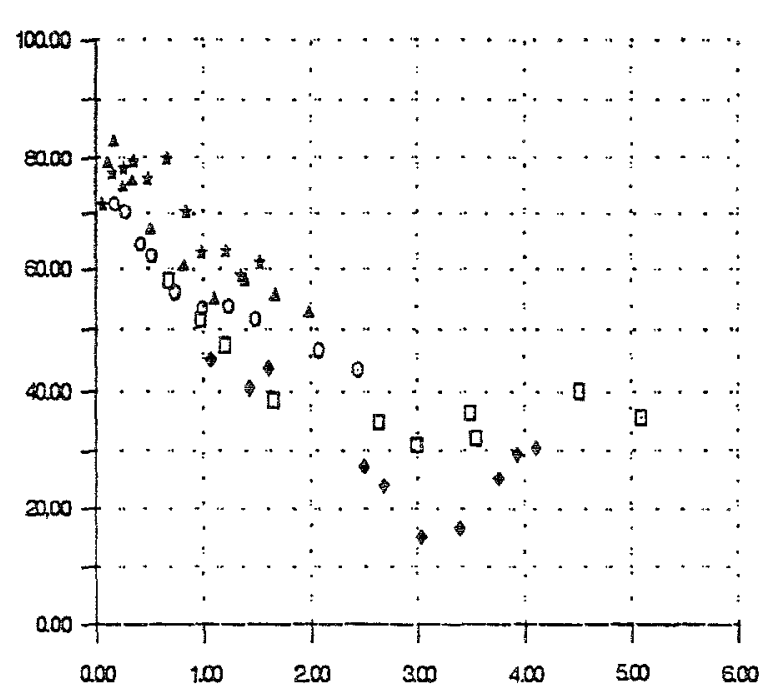

$\begin{array}{lll}T=0,6 s & T=1,6 s \quad 0 \\ T=1,1 s \quad \square & T=1,9 s \\ T=2,4 s & \end{array}$

chambre d'expansion de $60 \mathrm{~cm}$

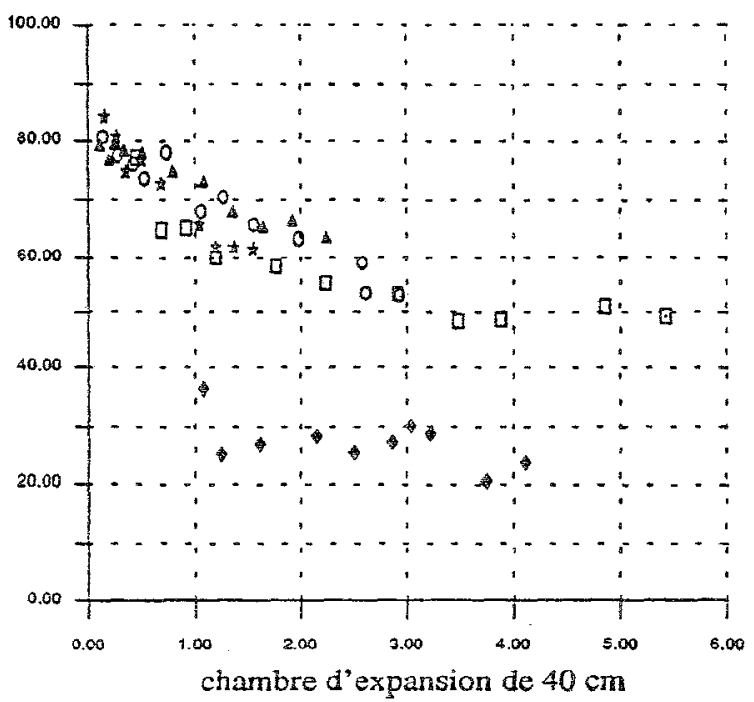




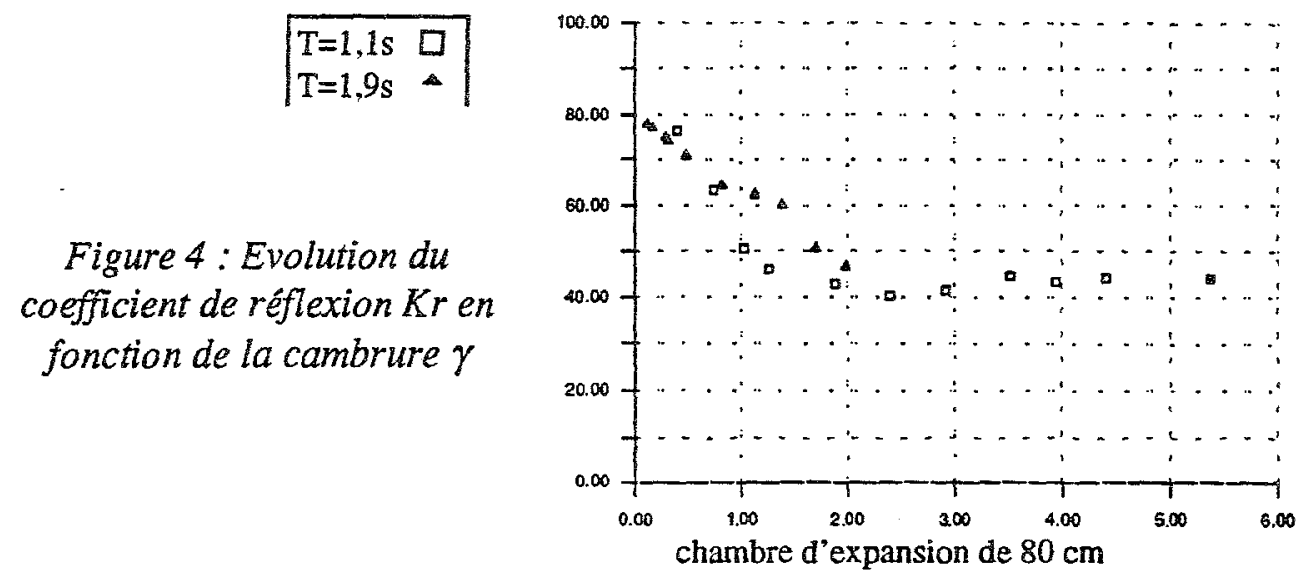

Les meilleurs résultats sont obtenus pour une largeur de chambre de $60 \mathrm{~cm}$ et atteignent 30 à $40 \%$ qui sont des valeurs tout à fait comparables à celles obtenues pour des caissons Jarlan dans les mêmes conditions.

Pour la période courte de 0,6 seconde, d'une longueur d'onde de $50 \mathrm{~cm}$, on constate une chute spectaculaire du coefficient de réflexion qui atteint 15 à $20 \%$ seulement.

Une analyse fine du phénomène a pu être faite en mesurant à l'intérieur de la chambre la cote de la surface libre au moment de l'arrivée d'un creux ou d'une crête pour les périodes de 0,6 et de $1,1 \mathrm{~s}$.

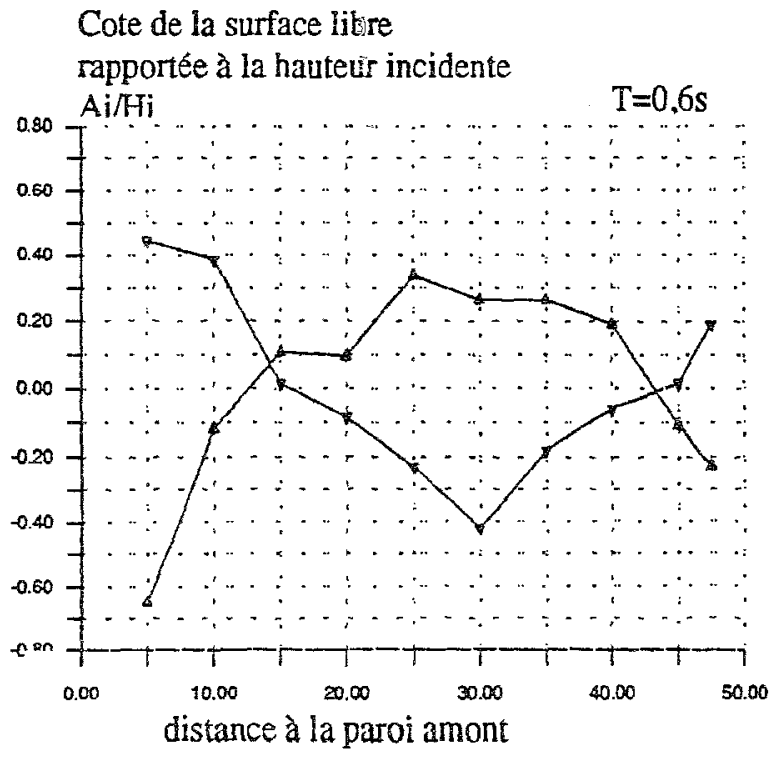


Figure 5 : Etat de la surface

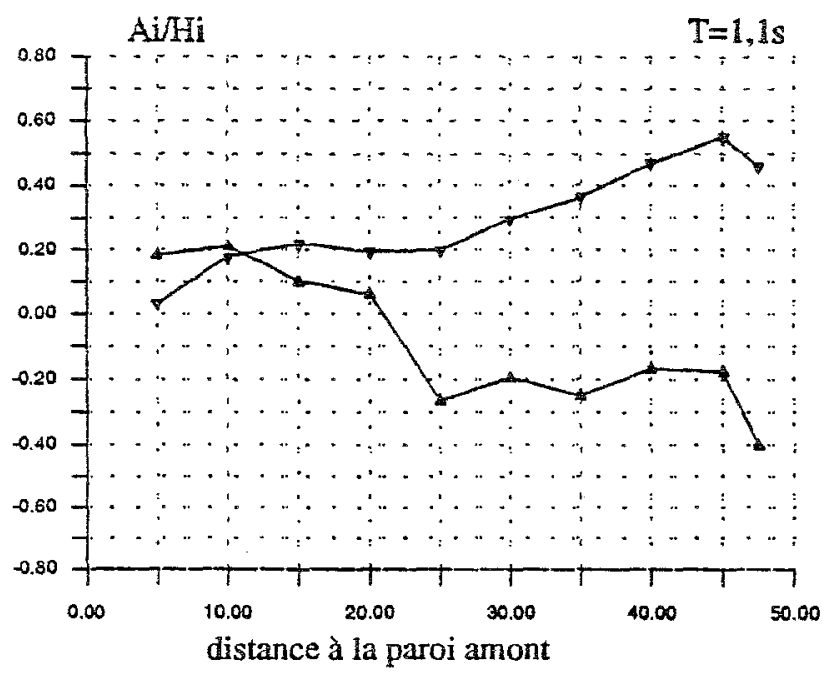

Pour la période de $0,6 \mathrm{~s}$ on observe un déphasage entre les deux cotes de la paroi perméable qui est de nature à expliquer le phénomène : pour la configuration testée la période de la houle de $0,6 \mathrm{~s}$. apparaît comme une période fondamentale de la structure.

\subsection{Lattes alternées}

Les résultats obtenus (Figure 6), montrent que malgré une modification importante de la face perméable, le comportement du système n'est pas fondamentalement modifié : les écarts avec la structure précédente (chambre de $60 \mathrm{~cm}$ ) ne dépassent pas $10 \%$.

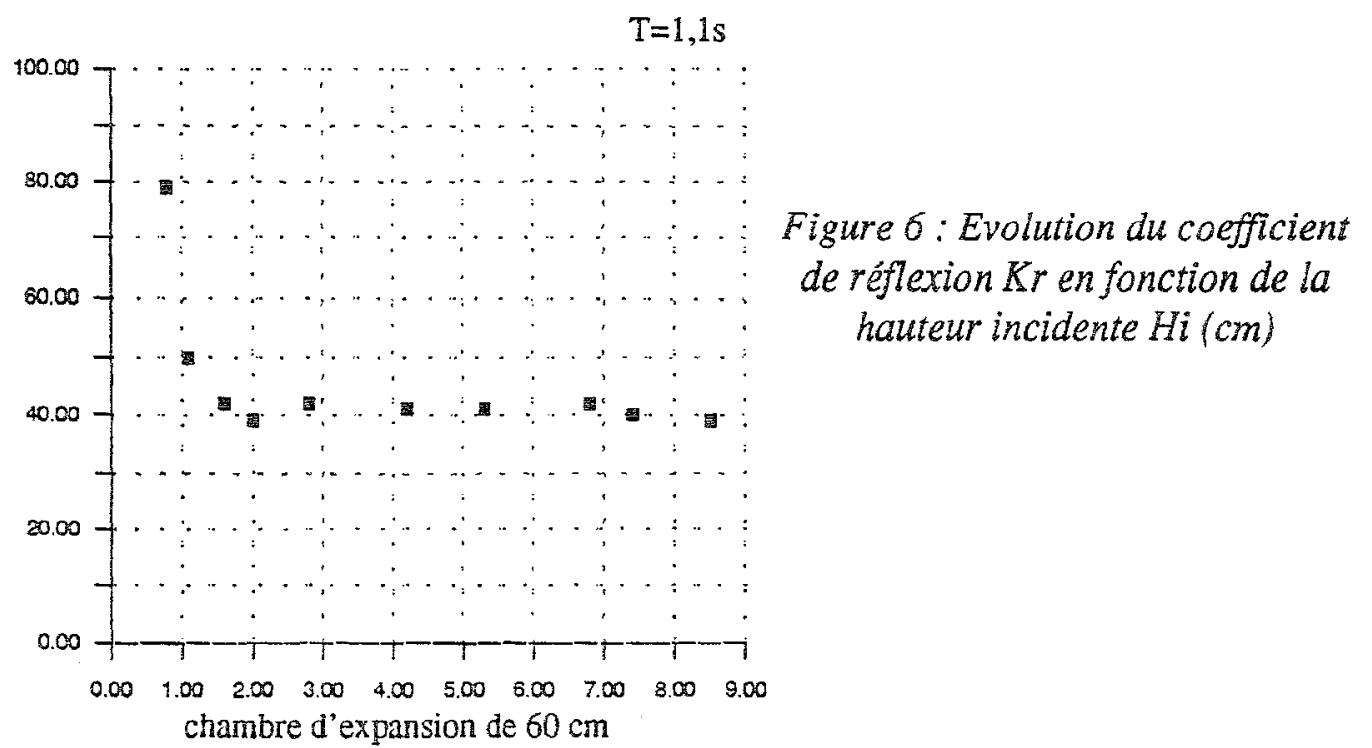




\section{Conclusion}

Les essais partiels qui ont été réalisés ont permis de caractériser le comportement des systèmes à persiennes et permettent de les classer dans la grande famille des digues verticales à parois perméables pour lesquelles on note :

-des périodes (ou des fréquences) de la houle pour lesquelles la structure a un comportement optimal.

-une faible influence de la géométrie de la paroi amont, à partir du moment où la configuration est correcte (porosité réelle suffisante ...).

II ne semble pas qu'un tel système puisse répondre aux exigences des plages d'amortissement pour lesquelles on attend des coefficients de réflexion ne dépassant pas 5 à $10 \%$ pour les faibles cambrures.

\section{Références}

CAMINADE, D.

«Etude de la transmission de la houle à travers une paroi perforée »

Thèse, université du Havre 1992, 163 p.

SZKLAREK, $P$.

«Etude du fonctionnement hydraulique d'un caisson Jarlan »

Rapport de stage de D.E.A. de génie civil, Université du Havre 1992, 58 p.

CAMINADE, D ARSIE, A.

«Evaluation de l'efficacité d'un dispositif d'amortissement de la houle de type persiennes »

Rapport de contrat $n^{\circ}$ R096, Groupe de Recherche en Génie Civil, Université du Havre 1994 